\title{
A New Generalisation of Sam-Solai's Multivariate symmetric Arcsine Distribution of Kind-1*
}

\author{
${ }^{1}$ Dr. G.S. David Sam Jayakumar. ${ }^{2}$ Dr.A.Solairaju. ${ }^{3}$ Mr.S.Dawood Ali \\ 1,3 (Assistant Professor,Jamal Institute of Management,Tiruchirappalli- 620020 South India, India.) \\ ${ }^{2}$ (Associate Professor, Dept.of mathematics, JamalMohamed,college,,Tiruchirappalli-620 020 South \\ India, India.)
}

\begin{abstract}
This paper proposed a new generalization of family of Sarmanov type Continuous multivariate symmetric probability distributions. More specifically the authors visualize a new generalization of Sam-Solai's Multivariate symmetric arcsine distribution of Kind-1 from the univariate case. Further, we find its Cumulation, Marginal, Conditional distributions, Generating functions and also discussed its special case.The special cases include the transformation of Sam-solai's Multivariate symmetric arcsine distribution of kind-1 into Multivariate symmetric log arcsine distribution of kind-1 and Multivariate symmetric Inverse arcine distribution of Kind-1. It is found that the conditional variance of Sam-Solai's Multivariate conditional symmetric arcsine distribution is homoscedastic and the correlation co-efficient among the random variables are similar to Pearson's product moment correlation co-efficient. Finally area values are obtained for Bi-variate symmetric arcsine distribution of kind-1 and Bivariate probability surfaces and contours are visualized.
\end{abstract}

Keywords: Sam-Solai's Multivariate symmetric arcsine distribution of kind-1, homoscedastic, Multivariate symmetric log arcsine distribution of kind-1, Multivariate symmetric Inverse arcsine distribution of Kind-1.

\section{Introduction}

The introduction of trigonometric distributions in the statistical literature is not unique; many authors studied the distribution before three decades.Trignometric functions are the age-old concepts and the application of these in mathematics and other fields are diverse. But the entrance and usage of trigonometric functions in distributions literature is little. Many authors studied and proposed new form of trigonometric distributions and let we see some of the pioneers. Norton [1975, 1978] proposed the arcsine law and later elaborately studied the moment properties of the distribution. Similarly Arnold et al [1980] also investigated some properties of the arcsine distribution and Tak'acs[1981] discussed the arcsine law proposed by the paul L'evy. On the other hand, Kemperman et al [1982] visualized the characterization of the arcsine distribution as an interesting property and Michealwoodroofe [1982] applied the arcsine law for the model selection purpose.Moreover,Kotz at al [1982],Cambanis et al [1983],Nadarajah et al[2006] were proposed the circular normal distributions, $\alpha$ symmetric distributions,Beta-Trignometric distributions which includes the sin and arcsine distributions are special cases respectively.Finally,as per the recent review,Mardia [2008,2011]studied the Von-mises distribution and mixtures of multivariate sine distributions with reference to the applications to bio-informatics. From the above discussed reviews, it is clear; most of the authors proposed and studied the univariate sinearcsine trigonometric distributions, except some authors. This motivates the authors of this paper to propose a new form of multivariate generalization of arcsine distribution. The structure and the properties of the multivariate arcsine distribution is discussed in the next and subsequent sections.

\section{II.Sam-Solai's Multivariate symmetric arcsine Distribution}

Definition 2.1: Let $X_{1}, X_{2}, X_{3}, \mathrm{~K} X_{q}$ are the random variables followed Continuous univariate symmetric arcsine distribution with mean 0 and variance (1/2) for all $\mathrm{i}$ ( $\mathrm{i}=1$ to $\mathrm{p}$ ). Then the density of Multivariate Sam-Solai's symmetric arcsine distribution of Kind- 1 is defined as

$$
\begin{gathered}
f\left(x_{1}, x_{2}, x_{3} \mathrm{~K}, x_{p}\right)=\left\{1+2 \underset{i=1}{\stackrel{p}{a}} \underset{j=1}{\stackrel{p}{a}} r_{i j} x_{i} x_{j}\right\}\left(\frac{1}{p}\right)^{p} \underset{i=1}{\stackrel{p}{\mathrm{O}}} \frac{1}{\sqrt{1-x_{i}^{2}}} \\
\text { where } i^{1} j-1 £ x_{i} £+1-1 £ r_{i j} £+1
\end{gathered}
$$

Theorem2.2: -The cumulative distribution function of the Sam-Solai's Multivariate symmetric arcsine distribution is defined by 


$$
\begin{aligned}
& F\left(Z_{1}, Z_{2}, Z_{3} \ldots, Z_{p}\right)=\int_{-1}^{z_{1}} \int_{-1}^{Z_{2}} \int_{-1}^{z_{3}} \ldots \int_{-1}^{z_{p}}\left\{1+2 \sum_{i=1}^{p} \sum_{j=1}^{p} r_{i j} u_{i} u_{j}\right\}\left(\frac{1}{\pi}\right)^{p} \prod_{i=1}^{p} \frac{1}{\sqrt{1-u_{i}^{2}}} d u_{i} \\
& \text { where }{ }_{i}{ }^{1} j-1 £ u_{i} £ Z_{i}-1 £ r_{i j} £+1 \\
& F\left(Z_{1}, Z_{2}, Z_{3} \ldots, Z_{p}\right)=\int_{-1}^{z_{1}} \int_{-1}^{z_{2}} \int_{-1}^{z_{3}} \ldots \int_{-1}^{z_{p}}\left(\frac{1}{\pi}\right)^{p} \prod_{i=1}^{p} \frac{1}{\sqrt{1-u_{i}^{2}}} d u_{i}+\int_{-1}^{z_{1}} \int_{-1}^{z_{2}} \int_{-1}^{z_{3}} \ldots \int_{-1}^{z_{p}}\left(2 \sum_{i=1}^{p} \sum_{j=1}^{p} r_{i j} u_{i} u_{j}\right)\left(\frac{1}{\pi}\right)^{p} \prod_{i=1}^{p} \frac{1}{\sqrt{1-u_{i}^{2}}} d u_{i} \\
& F\left(Z_{1}, Z_{2}, Z_{3} \mathrm{~K}, Z_{p}\right)=\underset{\mathrm{O}}{\mathrm{D}}\left(\frac{1}{2}+\frac{\arcsin \left(Z_{i}\right)}{p}\right)\left\{1+2 \mathrm{a}_{i=1}^{p} \underset{j=1}{\stackrel{p}{\mathrm{a}}} \frac{\left(r_{i j} \sqrt{1-Z_{i}^{2}} \sqrt{1-Z_{j}^{2}}\right) / p^{2}}{\left(\frac{1}{2}+\frac{\arcsin \left(Z_{i}\right)}{p}\right)\left(\frac{1}{2}+\frac{\arcsin \left(Z_{j}\right)}{p}\right)}\right\}
\end{aligned}
$$

Theorem2.3: The Probability density function of Sam-Solai's Multivariate symmetric arcsine distribution of $X_{1}$ on $X_{2}, X_{3}, \mathrm{~K} X_{P}$ is

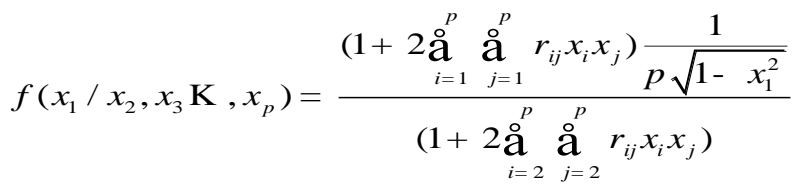

$$
\begin{aligned}
& \text { where } i^{1} \quad j-1 £ x_{1} £+1-1 £ r_{i j} £+1
\end{aligned}
$$

Proof: It is obtained from

$$
f\left(x_{1} / x_{2}, x_{3} \mathrm{~K}, x_{p}\right)=\frac{f\left(x_{1}, x_{2}, x_{3} \mathrm{~K}, x_{p}\right)}{f\left(x_{2}, x_{3} \mathrm{~K}, x_{p}\right)}
$$

Theorem 2.4- Even order conditional moments of Sam-Solai's Multivariate Conditional symmetric arcsine distribution is

$$
E\left(x_{1}^{2 n} / x_{2}, x_{3} \mathrm{~K}, x_{p}\right)=\frac{\mathrm{G}\left(n+\frac{1}{2}\right)}{\sqrt{p} \mathrm{G}(n+1)}
$$

Proof: The odd moments of Multivariate Conditional symmetric arcsine distribution are zero and the even order moments are given as

$$
\begin{aligned}
& E\left(x_{1}^{2 n} / x_{2}, x_{3} \mathrm{~K}, x_{p}\right)=\stackrel{\mathrm{o}}{\mathbf{O}}_{-1}^{+1} x_{1}^{2 n} f\left(x_{1} / x_{2}, x_{3} \mathrm{~K}, x_{p}\right) d x_{1}
\end{aligned}
$$

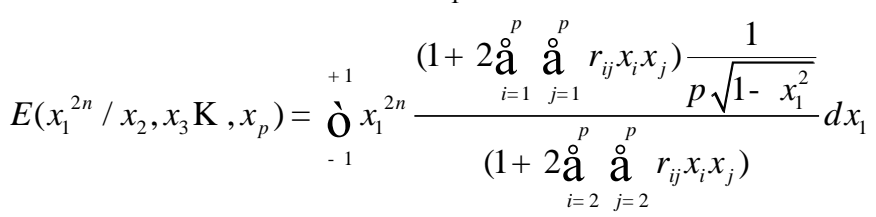

$$
\begin{aligned}
& E\left(x_{1}^{2 n} / x_{2}, x_{3} \mathrm{~K}, x_{p}\right)=\frac{\mathrm{G}\left(n+\frac{1}{2}\right)}{\sqrt{p} \mathrm{G}(n+1)}
\end{aligned}
$$

From (6) and if $n=1,2,3$, then the higher order conditional even moments of the distribution are

$$
\begin{aligned}
& E\left(x_{1}^{2} / x_{2}, x_{3} \mathrm{~K}, x_{p}\right)=\frac{1}{2} \\
& E\left(x_{1}^{4} / x_{2}, x_{3} \mathrm{~K}, x_{p}\right)=\frac{3}{8} \\
& E\left(x_{1}^{6} / x_{2}, x_{3} \mathrm{~K}, x_{p}\right)=\frac{5}{16}
\end{aligned}
$$


A New Generalisation Of Sam-Solai's Multivariate Symmetric Arcsine Distribution Of Kind-1*

Theorem2.5-If there are $\boldsymbol{p}=(\boldsymbol{q}+\boldsymbol{k})$ random variables, such that $\boldsymbol{q}$ random variables $X_{1}, X_{2}, X_{3}, \mathrm{~K} X_{q}$ conditionally depends on the $\boldsymbol{k}$ variables $X_{q+1}, X_{q+2}, X_{q+3}, \mathrm{~K} X_{q+k}$, then the density function of Sam-Solai's multivariate conditional symmetric arcsine distribution is

$$
\begin{aligned}
& f\left(x_{1}, x_{2}, x_{3} \mathrm{~K}, x_{q} / x_{q+1}, x_{q+2}, x_{q+3} \mathrm{~K}, x_{q+k}\right)=\frac{\left\{1+2 \stackrel{2+a}{a} \underset{i=1}{\stackrel{q}{\mathrm{a}}}{ }_{j=1}^{q+k} r_{i j} x_{i} x_{j}\right\}\left(\frac{1}{p}\right)^{q} \underset{i=1}{\mathrm{O}} \frac{1}{\sqrt{1-x_{i}^{2}}}}{q^{q+k} q^{q+k}} \\
& \left\{1+2 \underset{i=q+1}{\stackrel{\mathrm{a}}{\mathrm{a}}} r_{i j} x_{i} x_{j}\right\} \\
& \text { where } i^{1} \quad j-1 £ x_{i} £+1 \quad-1 £ r_{i j} £+1 \quad p=q+k
\end{aligned}
$$

Proof: Let the multivariate conditional law for $\boldsymbol{q}$ random variables $X_{1}, X_{2}, X_{3}, \mathrm{~K} X_{q}$ conditionally depends on the $\boldsymbol{k}$ variables $X_{q+1}, X_{q+2}, X_{q+3}, K X_{q+k}$ is given as

$$
\begin{aligned}
& f\left(x_{1}, x_{2}, x_{3}, \mathrm{~K} x_{q} / x_{q+1}, x_{q+2}, x_{q+3}, \mathrm{~K} x_{q+k}\right)=\frac{f\left(x_{1}, x_{2}, x_{3}, \mathrm{~K} x_{q}, x_{q+1}, x_{q+2}, x_{q+3}, \mathrm{~K} x_{q+k}\right)}{f\left(x_{q+1}, x_{q+2}, x_{q+3}, \mathrm{~K} x_{q+k}\right)}
\end{aligned}
$$

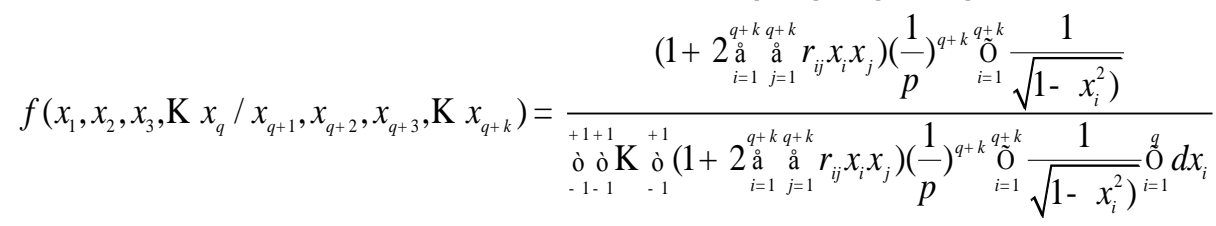

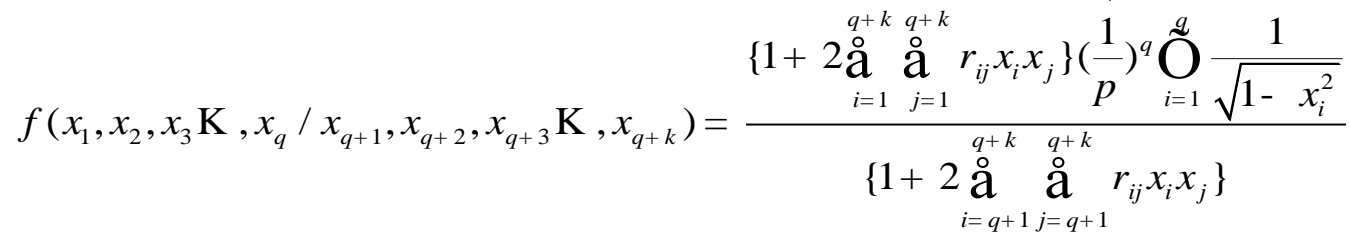

$$
\begin{aligned}
& \text { where } i^{1} \quad j-1 £ x_{i} £+1-1 £ r_{i j} £+1 \quad p=q+k
\end{aligned}
$$

\section{III.Constants of Sam-Solai's Multivariate symmetric arcsine Distribution}

Theorem 3.1 The Marginal Co-variance and Population Correlation Co-efficient between the random variables $X_{1}$ and $X_{2}$ is given as

$$
\operatorname{COV}\left(x_{1}, x_{2}\right)=\frac{r_{12}}{2}
$$

Proof: Let the product moment of the Sam-Solai's multivariate symmetric arcsine distribution in terms of Covariance from the origin is given as

$$
\begin{aligned}
& \operatorname{COV}\left(x_{1}, x_{2}\right)=\underset{-1-1}{+1+1} \mathbf{o ̀ ~}^{\mathrm{K}} \mathrm{O}_{-1}^{+1} x_{1} x_{2} f\left(x_{1}, x_{2}, x_{3}, \mathrm{~K} x_{p}\right) \stackrel{\mathrm{O}}{\mathrm{O}}_{i=1} d x_{i}
\end{aligned}
$$

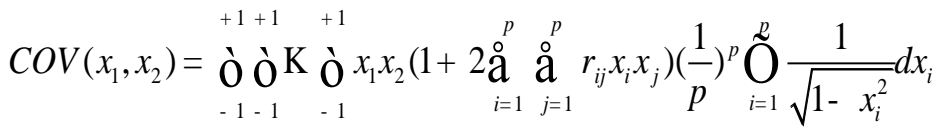

$$
\begin{aligned}
& \operatorname{COV}\left(x_{1}, x_{2}\right)=\frac{r_{12}}{2}
\end{aligned}
$$

Remark 3.1: The result can be generalized to the Co-variance between the $i^{\text {th }}$ and $j^{\text {th }}$ random variable are given as

$$
\begin{gathered}
\operatorname{COV}\left(x_{i}, x_{j}\right)=\frac{r_{i j}}{2} \\
r_{i j}=2 \operatorname{COV}\left(x_{i}, x_{j}\right) \\
\text { where } i^{1} j \quad-1 \notin r_{i j} \notin+1
\end{gathered}
$$

Theorem 3.2: The Moment generating function of Sam-Solai's Multivariate symmetric arcsine distribution is 


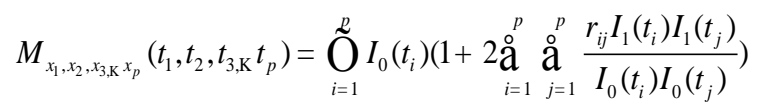

where $I_{0}\left(t_{i}\right)$ and $I_{1}\left(t_{i}\right)$ are the modified Bessel functions.

Proof: Let the moment generating function of the Multivariate distribution is given as

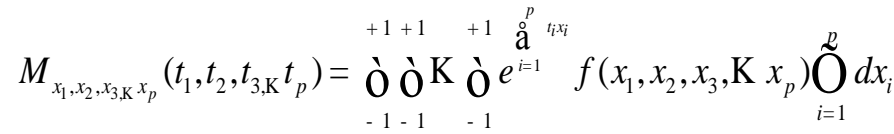

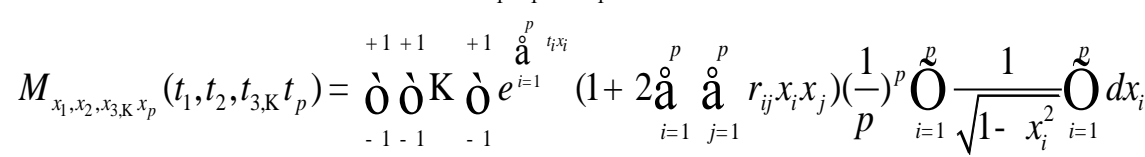

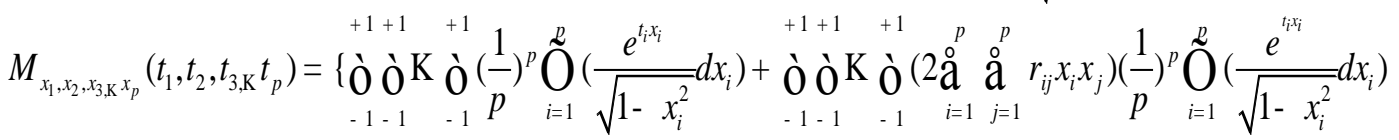

$$
\begin{aligned}
& \grave{\mathrm{O}}_{-1}^{+1} e^{t_{i} x_{i}} \frac{1}{p \sqrt{1-x_{i}^{2}}} d x_{i}=I_{0}\left(t_{i}\right) \\
& \grave{\mathrm{O}}_{-1}^{+1} x_{i} e^{t_{i} x_{i}} \frac{1}{p \sqrt{1-x_{i}^{2}}} d x_{i}=I_{1}\left(t_{i}\right)
\end{aligned}
$$

From (9), (10), (11) and by integration

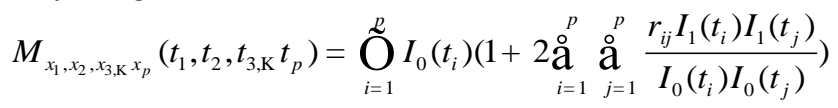

Theorem 3.3: The Cumulant of the Moment generating function of the Sam-Solai's Multivariate symmetric arcsine distribution is

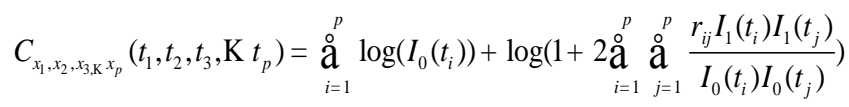

where $I_{0}\left(t_{i}\right)$ and $I_{1}\left(t_{i}\right)$ are the modified Bessel functions.

Proof: It is found from

$$
C_{x_{1}, x_{2}, x_{3, \mathrm{~K}} x_{p}}\left(t_{1}, t_{2}, t_{3, \mathrm{~K}} t_{p}\right)=\log \left(M_{x_{1}, x_{2}, x_{3, \mathrm{~K}} x_{p}}\left(t_{1}, t_{2}, t_{3, \mathrm{~K}} t_{p}\right)\right)
$$

Theorem 3.4: The Characteristic function of the Sam-Solai's Multivariate symmetric arcsine distribution is

$$
f_{x_{1}, x_{2}, x_{3, K} x_{p}}\left(t_{1}, t_{2}, t_{3, \mathrm{~K}} t_{p}\right)=\bigodot_{i=1}^{D} J_{0}\left(t_{i}\right)\left(1+2 \grave{a}_{i=1}^{p} \stackrel{\mathrm{a}}{j=1}_{j=1}^{p} \frac{r_{i j} J_{1}\left(t_{i}\right) J_{1}\left(t_{j}\right)}{J_{0}\left(t_{i}\right) J_{0}\left(t_{j}\right)}\right)
$$

where $J_{0}\left(t_{i}\right)$ and $J_{1}\left(t_{i}\right)$ are the Bessel functions.

Proof: Let the characteristic function of a multivariate distribution is given as

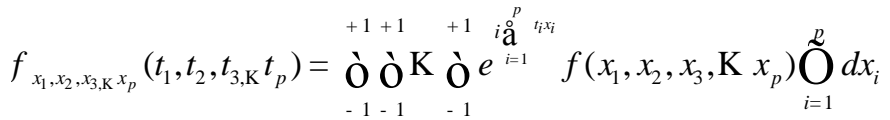

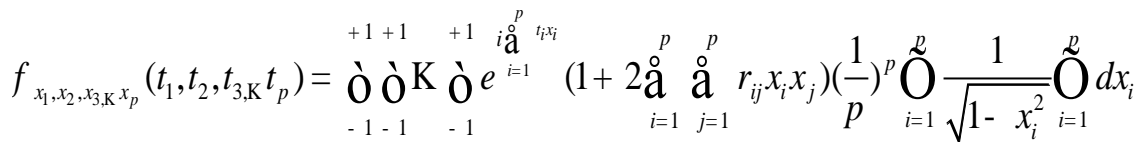

$$
\begin{aligned}
& f_{x_{1}, x_{2}, x_{3}, x_{p}}\left(t_{1}, t_{2}, t_{3, \mathrm{~K}} t_{p}\right)=\left\{\begin{array}{l}
+1+1 \\
\mathbf{O}
\end{array}\right. \\
& \grave{\mathrm{O}}_{-1}^{+1} e^{i i_{i} x_{i}} \frac{1}{p \sqrt{1-x_{i}^{2}}} d x_{i}=J_{0}\left(t_{i}\right) \\
& \grave{\mathrm{O}}_{-1}^{+1} x_{i} e^{i t_{i} x_{i}} \frac{1}{p \sqrt{1-x_{i}^{2}}} d x_{i}=J_{1}\left(t_{i}\right)
\end{aligned}
$$

From (14), (15), (16) and by integration

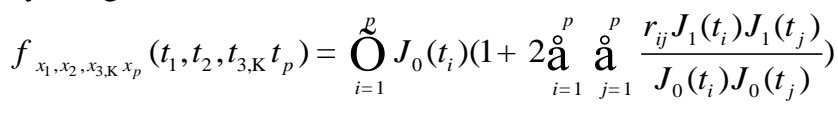




\section{IV.Some Special Cases}

Result 4.1: From (1) and if $r_{i j}=0$, then there is no correlation between the random variables and the SamSolai's multivariate symmetric arcsine density is reduced into product of the density function of uni-variate symmetric arcsine distributions.

Result 4.2: From (1) and if $\boldsymbol{P}=\mathbf{2}$, then the density of Sam-Solai's Multivariate symmetric arcsine distribution was reduced into

$$
\begin{gathered}
f\left(x_{1}, x_{2}\right)=\left(1+2 r_{12} x_{1} x_{2}\right) \frac{1}{p \sqrt{1-x_{1}^{2}}} \frac{1}{p \sqrt{1-x_{2}^{2}}} \\
\text { where }-1 £ x_{1} £+1-1 £ x_{2} £+1-1 £ r_{12} £+1
\end{gathered}
$$

\section{This is called Sam-Solai's Bi-variate symmetric Arcsine distribution of Kind-1}

Result 4.4: Below the diagram shows the Bi-variate probability surface of the Sam-Solai's Bi-variate standard symmetric arcsine distribution for various values of population correlation coefficient ( $r_{12}=-1,-0.75,-0.5,-0.25$, $0,+0.5,+0.25,+0.75+1)$ are given.

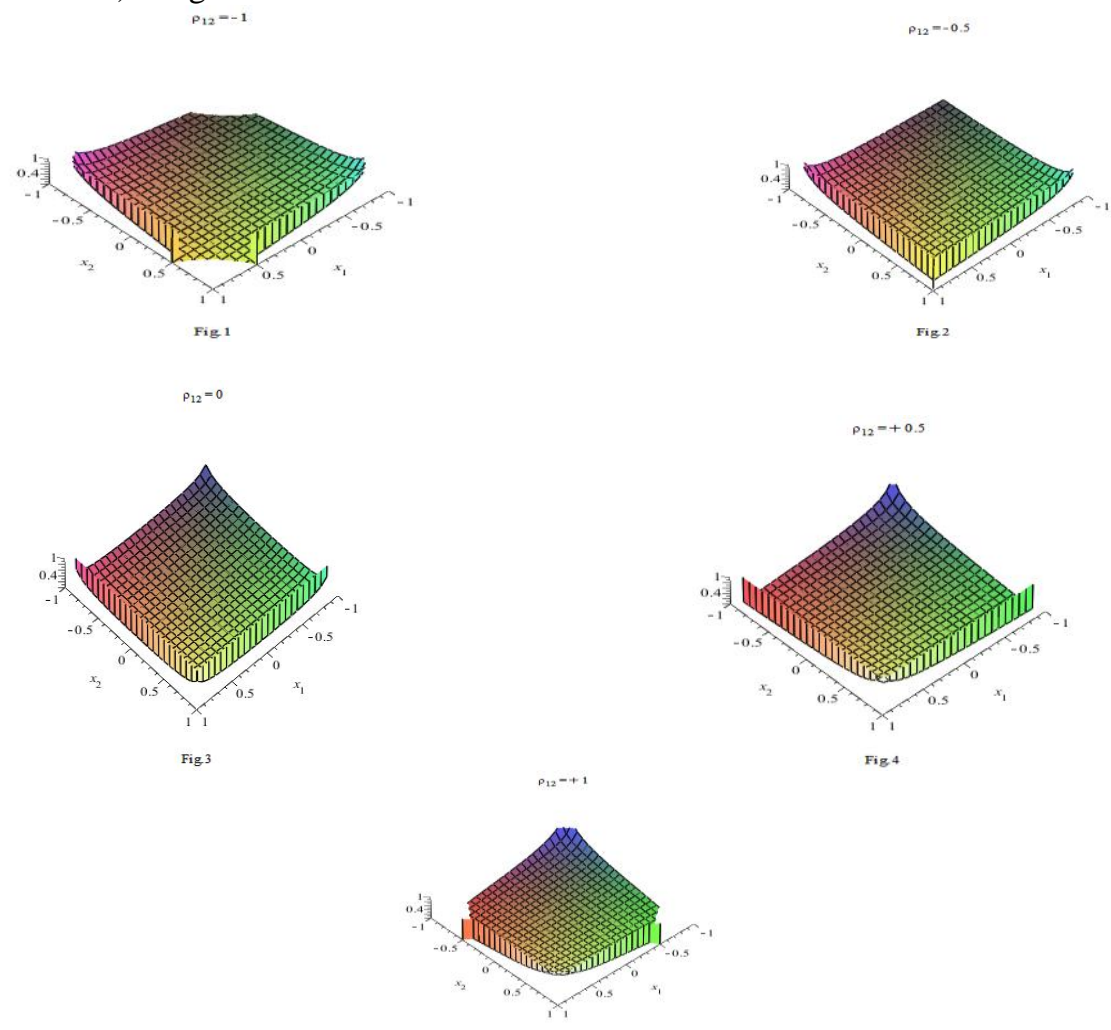

Result 4.5: From (2) and if $\boldsymbol{P}=\mathbf{2}$, then the Sam-Solai's Bi-variate Cumulative standard symmetric arcsine distribution is given as

$$
\left.F\left(Z_{1}, Z_{2}\right)=\left(\frac{1}{2}+\frac{\arcsin \left(Z_{1}\right)}{p}\right)\left(\frac{1}{2}+\frac{\arcsin \left(Z_{2}\right)}{p}\right)+2\left(r_{12} \sqrt{1-Z_{1}^{2}} \sqrt{1-Z_{2}^{2}}\right) / p^{2}\right)
$$

Result 4.6: Below the diagram shows the Bi-variate cumulative probability surface of the Sam-Solai's Bivariate cumulative symmetric arcsine distribution for various values of population correlation coefficient $\left(r_{12}=-\right.$ $1,-0.75,-0.5,-0.25,0,+0.25,+0.5,+0.75,+1)$ are given. 

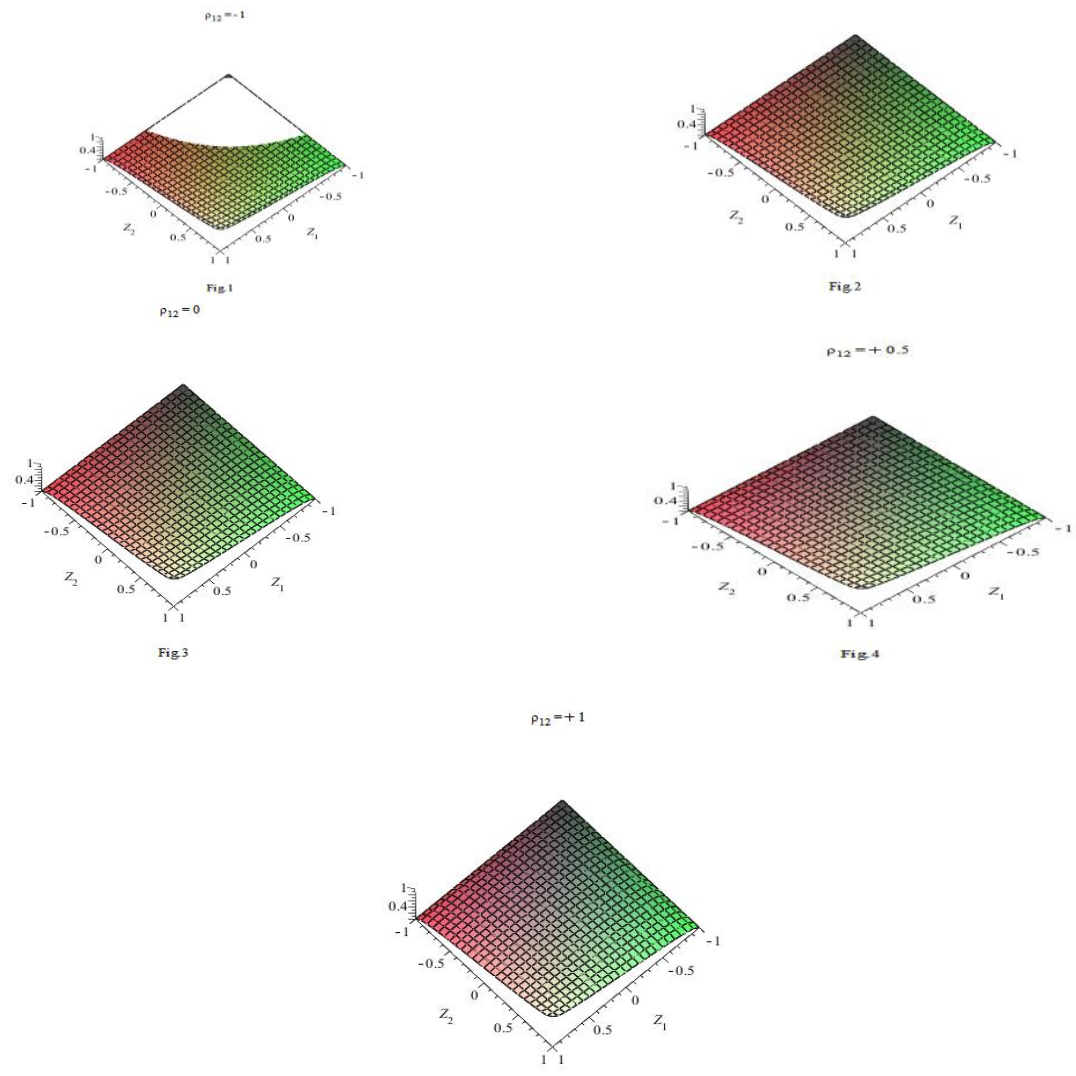

Result 4.7-Using the software Maple version 14, the table values from -0.9 to +0.9 with interval value 0.1 for Sam-Solai's bi-variate symmetric arcsine distribution are obtained. Area under the Sam-Solai's Bi-variate symmetric arcsine surfaces based on cumulative distribution function for various values of $r_{12}$ is given.

\begin{tabular}{|c|c|c|c|c|c|c|c|c|c|c|}
\hline \multirow{2}{*}{$Z_{1}$} & \multirow{2}{*}{$Z_{2}$} & \multicolumn{9}{|c|}{$r_{12}$} \\
\hline & & -1 & -0.75 & -0.5 & -0.25 & 0 & +0.25 & +0.5 & +0.75 & +1 \\
\hline-0.9 & -0.9 & -017792 & -.008174 & .001443 & .011061 & .020679 & .030297 & .039914 & .049532 & .059150 \\
\hline-0.8 & -0.8 & -.030856 & -.012633 & .005590 & .023813 & .042036 & .060259 & .078483 & .096706 & .114929 \\
\hline-0.7 & -0.7 & -.039080 & -.013264 & .012552 & .038368 & .064184 & .090000 & .115816 & .141633 & .167449 \\
\hline-0.6 & -0.6 & -.042383 & -.009987 & .022410 & .054807 & .087203 & .119600 & .151997 & .184394 & .216790 \\
\hline-0.5 & -0.5 & -040675 & -.002710 & .035255 & .073220 & .111184 & .149149 & .187114 & .225079 & .263044 \\
\hline-0.4 & -0.4 & -.033850 & .008670 & .051191 & .093712 & .136232 & .178753 & .221274 & .263794 & .306315 \\
\hline-0.3 & -0.3 & -.021785 & .024279 & .070343 & .116407 & .162471 & .208535 & .254599 & .300663 & .346727 \\
\hline-0.2 & -0.2 & -.004329 & .044266 & .092861 & .141456 & .190051 & .238646 & .287241 & .335836 & .384431 \\
\hline-0.1 & -0.1 & .018697 & .068811 & .118925 & .169038 & .219152 & .269266 & .319379 & .369493 & .419607 \\
\hline 0 & 0 & .047521 & .098141 & .148760 & .199380 & .250000 & .300620 & .351240 & .401859 & .452479 \\
\hline+0.1 & +0.1 & .082424 & .132538 & .182651 & .232765 & .282879 & .332992 & .383106 & .433219 & .483333 \\
\hline+0.2 & +0.2 & .123774 & .172369 & .220965 & .269560 & .318155 & .366750 & .415345 & .463940 & .512535 \\
\hline+0.3 & +0.3 & .172061 & .218125 & .264189 & .310253 & .356317 & .402381 & .448445 & .494509 & .540573 \\
\hline+0.4 & +0.4 & .227957 & .270477 & .312998 & .355519 & .398039 & .440560 & .483081 & .525601 & .568122 \\
\hline+0.5 & +0.5 & .292438 & .330403 & .368368 & .406333 & .444298 & .482263 & .520228 & .558193 & .596157 \\
\hline+0.6 & +0.6 & .367012 & .399409 & .431805 & .464202 & .496599 & .528996 & .561392 & .593789 & .626186 \\
\hline+0.7 & +0.7 & .454228 & .480044 & .505860 & .531676 & .557492 & .583308 & 609124 & .634940 & .660757 \\
\hline
\end{tabular}


A New Generalisation Of Sam-Solai's Multivariate Symmetric Arcsine Distribution Of Kind-1*

\begin{tabular}{|l|l|l|l|l|l|l|l|l|l|l|}
\hline \hline+0.8 & $+\mathbf{0 . 8}$ & .559089 & .577312 & .595535 & .613758 & .631981 & .650205 & .668428 & .6866651 & .704874 \\
\hline+0.9 & +0.9 & .694605 & .704223 & .713841 & .723458 & .733076 & .742694 & .752312 & .761929 & .771547 \\
\hline
\end{tabular}

Result 4.8: From (1) and if $y_{i}=e^{x_{i}}$, then the Sam-Solai's Multivariate symmetric arcsine distribution of Kind1 transformed into Sam-solai's Multivariate symmetric log arcsine distribution of Kind-1 and it's density is given as

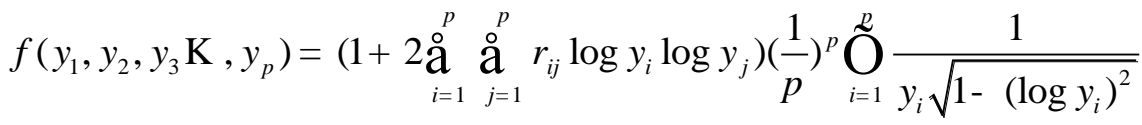

$$
\begin{aligned}
& \text { where } i^{1} \quad j \quad e^{-1} £ y_{i} £ e-1 £ r_{i j} £+1
\end{aligned}
$$

Result 4.9: From (2) and if $y_{i}=1 / x_{i}$, then the Sam-Solai's Multivariate symmetric arcsine distribution of Kind-1 transformed into Sam-solai's Multivariate symmetric Inverse arcsine distribution of Kind-1 and it's density is given as

$$
\begin{gathered}
f\left(y_{1}, y_{2}, y_{3} \ldots, y_{p}\right)=\left(1+2 \sum_{i=1}^{p} \sum_{j=1}^{p} \frac{r_{i j}}{y_{i} y_{j}}\right)\left(\frac{1}{\pi}\right)^{p} \prod_{i=1}^{p} \frac{1}{y_{i} \sqrt{y_{i}^{2}-1}} \\
\text { where } i^{1} j-1 £ y_{i} £+1-1 £ r_{i j} £+1
\end{gathered}
$$

\section{V.Conclusion}

The multivariate generalization of Sam-Solai's multivariate symmetric arcsine distribution of kind- 1 is having some interesting features. At first, the marginal uni-variate distributions of the Sam-Solai's Multivariate symmetric arcsine distributions are univariate symmetric arcsine distributions. Secondly, the Correlation coefficient of the proposed distribution are similar to Pearson's correlation Co-efficient. The Conditional variance of Sam-Solai's Multivariate Conditional symmetric arcsine distribution is heteroscedastic in nature and this feature is a unique for the proposed distribution. Thus the generalization of sarmonav family of symmetric multivariate distribution open the way for logical extension of the generalization of symmetric family of all univariate continuous probability distributions. Finally, the Multidimensional Jacobian transformation of Multivariate arcsine variables which leads us to generalize the Sam-solai's Multivariate symmetric log arcsine distribution and Multivariate symmetric Inverse arcsine distribution.

\section{References}

[1] M. Abramowitz and I. A. Stegun (Handbook of Mathematical Functions. Dover Publications, 1964)

[2] I.S. Gradshteyn and I.M. Ryzhik.(Table of integrals, series, and products. Academic Press, NewYork, 1965)

[3] R.M. Norton ,On properties of the arc sine law. Sankhya Ser. A, 37(2), 1975, 306-308

[4] R.M. Norton ,Moment properties and the arc sine law. Sankhya Ser. A, 40(2), 1978,192-198

[5] B.C. Arnold and R.A. Groeneveld, Some properties of the arcsine distribution. J.Amer. Statist.Assoc., 75(369), 1980,173-175.

[6] Tak'acs, L, The arc sine law of Paul L'evy. In Contributions to Probability. A Collection of Papers Dedicated to Eugene Luk'acs (J. Gani and V. K. Rohatgi, eds.) ,1981,49-63. Academic Press, New York

[7] J.H.B. Kemperman and M. Skibinsky, On the characterization of an interesting property of the arcsine distribution. Pacific J. Math., 103(2), 1982,457-465

[8] Michael Woodroofe, On Model Selection and the ARC Sine Laws, The Annals of Statistics. Volume 10, Number 4, 1982, 11821194.

[9] Kotz, S. and Johnson, N. L ,Circular normal distribution. Encyclopedia of Statistical Sciences, 1,479. 1982.

[10] S. Cambanis, R. Keener, and G. Simons ,On a $\alpha$-symmetric multivariate distributions.J. Multivariate Analysis, 13, 1983, $213-233$.

[11] Johnson, N. L., Kotz, S. and Balakrsihnan, N ,Continuous Univariate Distributions.1, 2nd ed., Wiley, New York, 1994.

[12] Nadarajah, S. and Kotz, S,Beta Trigonometric Distribution. Portuguese Economic Journal, Vol 5, No 3, 2006 , pp 207-224.

[13] K. V. Mardia, G. Hughes, C. C. Taylor, and H. Singh, A multivariate von Mises distribution with applications to bioinformatics. The Canadian Journal of Statistics,36(1), 2008,99-109

[14] K. V. Mardia, J. T. Kent, Z. Zhang, C. Taylor, and T. Hamelryck, Mixtures of concentrated multivariate sine distributions with applications to bio-informatics. Submitted, 2011.

[15] Solairaju, David Sam and Kavita Devi, A New Generalisation of Sam-Solai's Multivariate Laplace distribution,Global Journal of Mathematical sciences: Theory and Practical,(2012) Vol.4, Number 1, 2012, pp.63-80.

[16] Solairaju, David Sam and Kavita Dev, A New Generalisation of Sam-Solai's Multivariate-t-distribution, Global Journal of Mathematical sciences: Theory and Practical, Vol.4, Number 1, 2012, pp.81-95.

[17] Solairaju,David Sam and Kavita Devi, A New Generalisation of Sam-Solai's Multivariate Cauchy distribution of Type-I, Global Journal of Theoretical and applied Mathematical sciences:Vol.2, Number 1, 2012,pp.49-61.

[18] Solairaju, David Sam and KavitaDevi, A New Generalisation of Sam-Solai's Multivariate Cauchy distribution of Type-II, Global Journal of Theoretical and applied Mathematical sciences:Vol.2, Number 1, 2012,pp.63-75. 